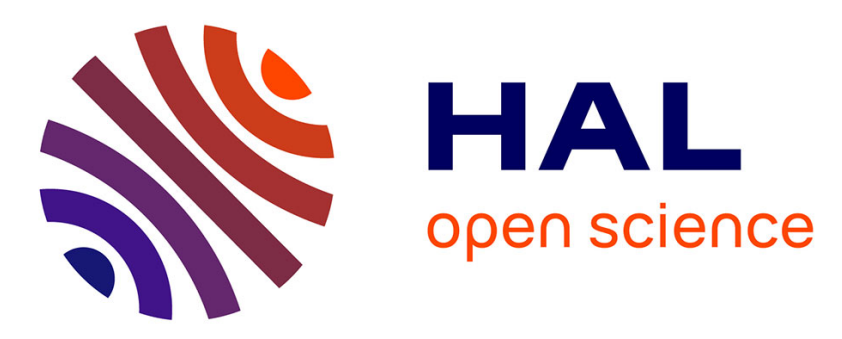

\title{
The $\alpha \delta$ method for modelling expert judgement and combination of non-destructive testing tools in risk-based inspection context: application to marine structures
}

\author{
Franck Schoefs, Jérôme Boéro, Alexandre Clément, Bruno Capra
}

\section{To cite this version:}

Franck Schoefs, Jérôme Boéro, Alexandre Clément, Bruno Capra. The $\alpha \delta$ method for modelling expert judgement and combination of non-destructive testing tools in risk-based inspection context: application to marine structures. Structure and Infrastructure Engineering, 2012, 8 (6), pp.531-543. 10.1080/15732479.2010.505374 . hal-01007359

\section{HAL Id: hal-01007359 \\ https://hal.science/hal-01007359}

Submitted on 16 Jun 2014

HAL is a multi-disciplinary open access archive for the deposit and dissemination of scientific research documents, whether they are published or not. The documents may come from teaching and research institutions in France or abroad, or from public or private research centers.
L'archive ouverte pluridisciplinaire HAL, est destinée au dépôt et à la diffusion de documents scientifiques de niveau recherche, publiés ou non, émanant des établissements d'enseignement et de recherche français ou étrangers, des laboratoires publics ou privés. 


\title{
The $\alpha \delta$ method for modelling expert judgement and combination of non-destructive testing tools in risk-based inspection context: application to marine structures
}

\author{
Franck Schoefs $^{\mathrm{a} *}$, Jérôme Boéro $^{\mathrm{b}}$, Alexandre Clément ${ }^{\mathrm{b}}$ and Bruno Capra ${ }^{\mathrm{b}}$ \\ ${ }^{a}$ Research Institute of Civil Engineering and Mechanics (GeM), UMR-CNRS 6183, Nantes Atlantic University, 2 rue de la \\ Houssinière, B.P. 92208, 44322 Nantes Cedex 03, France; ${ }^{b}$ Oxand S.A, 49 Avenue Franklin Roosvelt, 77210 Avon, France
}

\begin{abstract}
Owners of civil infrastructure base their maintenance decision schemes mainly on both structural integrity assessment and consequence analysis. Some inputs come from information collected by inspections with nondestructive or destructive tools. Uncertainties and errors of measurement can lead to bad decisions but are rarely integrated into the decision process. Currently, risk-based inspection (RBI) provides the basic concepts for optimising the maintenance plans of existing structures while insuring satisfactory safety and availability of the structure during its service life. It relies both on reliability computations and probabilistic modelling of inspection results. This last point leads to introduce the probability of detection and the probability of false alarms that are usually considered as parameters in RBI. However, when data from inter-calibration campaigns are available, the whole receiver operating characteristics curves should be used. Then, the comparison of non-destructive testing tools in terms of cost or benefit is difficult as well as the method for optimising a given technique. This article presents the $\alpha \beta$ method that gives a new performance indicator in this context. It is applied to the field of inspection of harbour structures.
\end{abstract}

Keywords: decision process; expert judgement; harbour structures; non-destructive testing (NDT) tools; receiver operating characteristic (ROC) curve; risk-based inspection (RBI)

\section{Introduction}

Replacement of engineering structures results in high economic and environmental costs, thus increasing the interest in maintaining these structures with efficient management plans. Therefore, the challenge for the owners consists of guaranteeing the operation and safety of ageing structures, while ensuring the reasonable costs and the availability conditions. Harbour structures meet all these stakes.

Reassessment of existing structures generates a need for updated material properties. In a lot of cases, on-site inspections are necessary and in some cases visual inspections are not sufficient. Non-destructive testing (NDT) tools are required for the inspection of coastal and marine structures where marine growth acts as a mask or where immersion gives poor conditions for inspection (visibility,..., etc.). In these fields, the cost of inspection can be prohibitive and an accurate description of the on-site performance of NDT tools must be provided. Inspection of existing structures by a NDT tool is not perfect and it has become a common practice to model their reliability in terms of probability of detection (PoD), probability of false alarms (PFA) and receiver operating characteristic (ROC) curves (Rouhan 2001, Rouhan and Schoefs 2003, Straub and Faber 2003, Pakrashi et al. 2008). These quantities are generally the main inputs needed by owners of structures who are looking to achieve Inspection, Maintenance and Repair plans (IMR) (Sheils et al. 2008) through risk-based inspections (RBI) methods. The assessment of PoD and PFA is even deduced from inter-calibration of NDT tools or from the modelling of the noise and the signal (Barnouin et al. 1993, Rudlin 1996, Rudlin and Dover 1996). Both because of their great economic interest and the cost (direct and indirect) of inspection, authors have selected steel harbour structures for the application.

First, on the basis of several previous works, this article reviews the theoretical aspects arising from detection theory and probabilistic modelling of inspections results. The objective is to provide inputs in the computation of mathematical expectation of RBI cost models. Methods for the combination of multiple inspections are also detailed. It is shown how these models highlight the role of the probability of defect presence. Expert judgement or the knowledge of ageing laws allows quantifying this probability (Rouhan and Schoefs 2003). The effects of the cost model and the

\footnotetext{
*Corresponding author. Email: franck.schoefs@univ-nantes.fr
} 
combination of multiple inspections are also highlighted.

In the second section, the article focuses on the use of the whole ROC curve in the case where no intercalibration campaign is available. A parametric modelling is used to assess the effect of the shape of ROC curves on the decision process. The article also introduces the polar coordinates of NDT-BPP for characterising ROC curves, which allows the parametric studies to perform.

Finally, this article focuses on the benefit of the combination of multiple non-destructive tests and the role of expert judgement in the cost-based decision after obtaining results from two inspections. The interest of such a combination appears specifically when one technique leads to a good PoD and a very fair PFA when another one leads to a very good PoD and a non-negligible PFA.

These concepts are applied on the RBI of steel harbour structures submitted to uniform corrosion (steel sheet pile seawalls).

\section{Probabilistic modelling of inspection based on detection theory}

\subsection{Probability of detection and probability of false alarm}

The most common concept which characterises inspection tool performance is the PoD. Let $a_{\mathrm{d}}$ be the minimal defect size, under which it is assumed that no detection is done. Parameter $a_{\mathrm{d}}$ is called detection threshold. Thus, the PoD is defined as (Equation (1)):

$$
\mathrm{PoD}=P\left(\hat{d} \geq a_{\mathrm{d}}\right)
$$

where $\hat{d}$ is the measured defect size.

Let's assume that the noise and the signal amplitude are independent random variables, then PoD and PFA have the following expression (Equations (2) and (3)):

$$
\begin{aligned}
& \text { PoD }=\int_{a_{\mathrm{d}}}^{+\infty} f_{\text {signal }}(\hat{d}) \partial \hat{d} \\
& \text { PFA }=\int_{a_{\mathrm{d}}}^{+\infty} f_{\text {noise }}(\eta) \partial \eta
\end{aligned}
$$

where $\hat{d}$ is the measured response level of NDT tool, i.e. 'signal + noise', $f_{\text {signal }}$ and $f_{\text {noise }}$ are respectively the probability density functions (pdfs) of 'signal + noise' (or measured defect) and 'noise'.

Thus, the PoD is a function of the detection threshold, the defect size and the noise, while the PFA depends on the detection threshold and the noise only (Schoefs et al. 2008b). Noise is because of the decision- chain 'physical measurement-decision on defect measurement transfer of information', the harsh environment of inspection and the complexity of testing procedure (link diver-inspector).

Figure 1 illustrates the pdf and the area to be computed for the evaluation of PoD and PFA for a given detection threshold in the case where 'signal + noise' and 'noise' are normally distributed.

\subsection{Receiver operating characteristic curve}

The ROC curve links the PoD and the PFA. For a given detection threshold, the couple (PFA, PoD) allows defining NDT performance. This couple can be considered as coordinates of a point in $R^{2}$ with axes representing PFA and PoD. Let us consider that $a_{\mathrm{d}}$ takes values in the range $[-\infty ;+\infty]$, these points belong to a curve called ROC which is a parametric curve with parameter $a_{\mathrm{d}}$ and defined by Equations (2) and (3).

The example of a ROC curve (ROC 3 ) plotted on Figure 2 is computed with the pdf presented on Figure 1 corresponding to normal distributions.

The ROC curve is a fundamental characteristic of the NDT tool performance for a given defect size. Perfect tool is represented by a ROC curve reduced to a single point whose coordinates are: $(\mathrm{PFA}, \mathrm{PoD})=$ $[0,1]$. A ROC curve represents a NDT tool performance facing a given pdf of a defect or a defect range. More details are available in Rouhan and Schoefs (2003).

Figure 2 presents six theoretical ROC curves, each one corresponding to different NDT tool performances. The worst curve is ROC 1, meaning that some noise can be easily detected, even if nothing is to be detected which finally leads to a high number of false alarms. As a result, overall performances are then poor. In contrast, the best plotted ROC curve is ROC

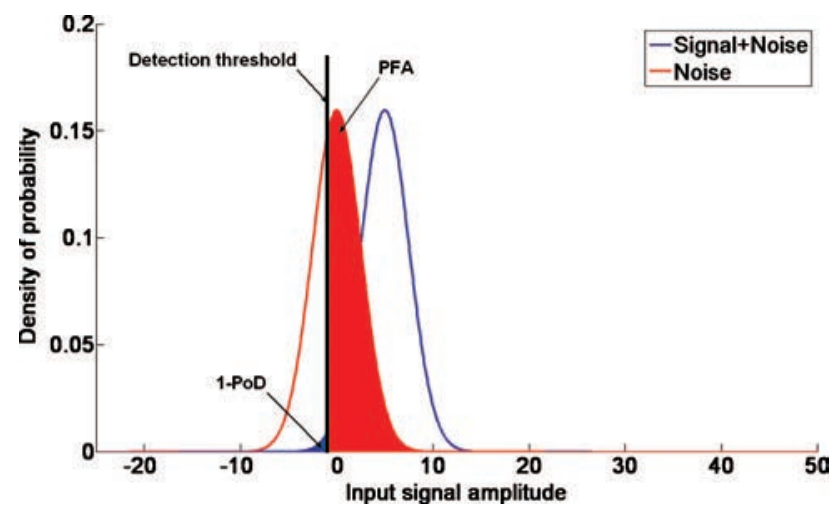

Figure 1. Illustration of PoD and PFA (signal + noise and noise normally distributed) for detection threshold $a_{\mathrm{d} 1}$. 


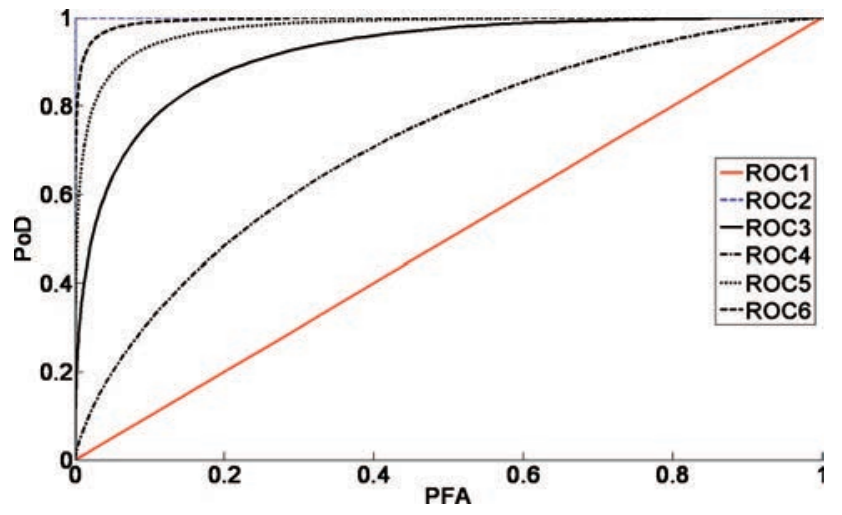

Figure 2. Example of ROC curves with several NDT performance.

2, which differs considerably with the previous curve. The PoD reaches very quickly to values near 1 , with small probabilities of false alarms for high values of PoD. Overall performances are very good. These ROC curves can be obtained by considering two techniques and the same defect range, one technique and two defect ranges, or one technique applied in various conditions (even if the testing procedure is rigorously followed during inspection). This is the case for underwater inspections of marine/coastal structures where accessibility and visibility are limited and conditions for the use of NDT tools are not optimal.

A simple geometric characterisation of ROC curves is the distance between the curve and the best performance point (BPP) of coordinates $(\mathrm{PFA}=0$, PoD = 1) (Schoefs and Clément 2004): by definition, the bigger the distance, the worst the performance. The corresponding point on the ROC curve is called the performance point of the NDT tool (NDT-BPP). As the configurations of ROC curves for the same distance are various, this article defines a curve characterisation by using the polar coordinates of the NDT-BPP. The NDT-BPP polar coordinates are defined by:

- the radius $\delta_{\mathrm{NDT}}$ equals the performance index (NDT-PI) (distance between the BPP and the ROC curve) (Schoefs and Clément 2004, Schoefs et al. 2007, 2008a);

- the $\alpha$ NDT is the angle between axis $(\mathrm{PFA}=0)$ and the line (BPP, NDT-BPP).

Assessment of PoD and PFA from the knowledge of detection threshold can be directly deduced from inter-calibration of NDT tools (Barnouin et al. 1993, Rudlin 1996, Rudlin \& Dover 1996). Generally, these projects are expensive, and consequently, it is sometimes necessary to choose another approach.
This article takes place in the context where intercalibration is not available. Calculation of PFA and PoD thereby results from probabilistic modelling of the 'noise' and 'signal + noise' pdf.

Predefined pdf in view of fitting 'noise' and 'signal + noise' distributions, or projection on polynomial chaos can be used (Schoefs et al. 2008b, 2009a). In this article, we choose to calculate PoD and PFA for the entire range of possible detection thresholds by the following expressions (4) and (5).

$$
\begin{aligned}
& \mathrm{PoD} \approx \frac{\operatorname{Card}(A)}{N_{\mathrm{d}}} ; A=\left\{j \in\left\{1, \ldots, N_{\mathrm{d}}\right\} ; d\left(\theta_{j}\right)>a_{\mathrm{d}}\right\} \\
& \mathrm{PFA} \approx \frac{\operatorname{Card}(B)}{N_{\eta}} ; B=\left\{j \in\left\{1, \ldots, N_{\varepsilon}\right\} ; \eta\left(\theta_{j}\right)>a_{\mathrm{d}}\right\}
\end{aligned}
$$

where $N_{\mathrm{d}}$ and $N_{\eta}$ are the number of 'signal + noise' and 'noise' measurements, respectively, and $\operatorname{Card}(\Theta)$ is the abbreviated form of cardinal, i.e. the number of elements in the mathematical set $\Theta$.

ROC curves are then plotted by connecting these points in the plan (PFA, PoD).

\section{Introduction of inspection in decision process}

\subsection{Bayesian modelling of inspection results}

To introduce the inspections results in a RBI scheme, conditional probabilities have to be introduced. In doing this, Bayesian modelling is then suitable and decision theory offers the theoretical basis (Rouhan and Schoefs 2003, Schoefs and Clément 2004).

Let us introduce four conditional probabilities:

- $P_{1}$ : probability that there is no defect present, conditional to no defect detection;

- $P_{2}$ : probability that there is no defect present, conditional to defect detection;

- $P_{3}$ : probability of the presence of defect, conditional to no defect detection;

- $P_{4}$ : probability of the presence of defect, conditional to defect detection.

In these definitions, the focus is on the presence or the absence of a defect after an inspection. After being calculated, the expressions of these probabilities as functions of PoD and PFA are deduced (Rouhan and Schoefs 2003) (see Equations (6)-(9)):

$$
\begin{gathered}
P_{1}=\frac{(1-\operatorname{PFA})(1-\gamma)}{(1-\operatorname{PoD}) \gamma+(1-\operatorname{PFA})(1-\gamma)} \\
P_{2}=\frac{\operatorname{PFA}(1-\gamma)}{\operatorname{PoD} \gamma+\operatorname{PFA}(1-\gamma)}
\end{gathered}
$$




$$
\begin{gathered}
P_{3}=\frac{(1-\mathrm{PoD}) \gamma}{(1-\mathrm{PoD}) \gamma+(1-\mathrm{PFA})(1-\gamma)} \\
P_{4}=\frac{\operatorname{PoD} \gamma}{\operatorname{PoD} \gamma+\operatorname{PFA}(1-\gamma)}
\end{gathered}
$$

where $\gamma$ is the probability of defect presence (see Equation (10)):

$$
P(X=1)=\gamma ; P(X=0)=1-\gamma
$$

In case of inspection of in-service structures, the defect presence is unknown. So $\gamma$ is defined by expert judgement or predictive ageing laws.

An illustration of $P_{2}$ as a function of PoD and PFA for a probability of defect presence of 0.1 is presented in Figure 3. When considering young structures, the low value of $\gamma=0.1$ can be understood as the probability of presence of large defects, and the high value of $\gamma=0.9$ as the probability of presence of small defects.

\subsection{Projection of ROC curves on $P_{i}$ surfaces}

In performing a cost analysis, points from the ROC curves can be considered for the computation of $P_{i\{i \in[1: 4]\}}$ (Rouhan and Schoefs 2003). If the detection threshold is unknown, the performance of the technique is given by the whole ROC curve. Then, we compute the average level of the projection of the ROC curves onto $P_{i}$ surfaces. Projection for two ROC curves on $P_{2}$ (ROC 4 and ROC 5) is plotted on Figure 3. The $P_{i}$ is then replaced in cost analysis by this geometric mean value (see Equation (11)):

$$
\mu\left(\left(P_{i}\right)_{\mid \mathrm{ROC}}\right)=\frac{1}{L_{\mathrm{ROC}}} \int_{\mathrm{ROC}} P_{i \mid \mathrm{ROC}} d l_{\mathrm{ROC}}
$$

where $l_{\text {ROC }}$ is the curvilinear abscissa along the projected ROC curve on $P_{i}$ surface, $P_{i \mid \text { ROC }}$ value of $P_{i}$ at this abscissa and $L_{\mathrm{ROC}}$ the length of ROC curve in (PFA, PoD) plane. This geometric mean value can be interpreted as the ratio of the area under the projected ROC and the length of this curve in the plane (PFA, PoD) presented in Figure 3.

\subsection{Cost analysis}

Optimisation of inspection plans generally involves the minimisation of a cost function. Seeing as this cost function is a random function, the problem is generally formulated as the minimisation of the total expected cost when performing scenarios of actions over a given period (Equation (12)).

$$
E(C)=\sum_{i} C\left(S_{i}\right) P\left(S_{i}\right)
$$

where $C\left(S_{i}\right)$ is the cost associated with the $i$ th scenario, $S_{i}$ and $P\left(S_{i}\right)$ is the probability that the $i$ th scenario occurs. Note that some studies also consider the effect of the policy on the scatter of the cost by computing, for example, the $p \%$ quantiles of the distribution of $C(S)$ (Schoefs et al. 2009b).

In case of inspections, two cost functions are considered. They are related to the cases of detection or no detection. The set of scenarios depends on the actions conditioned by the results of the inspection, termed maintenance policy in the following sections. For simplicity, the maintenance policy is:

- a 'detection' leads to a repair;

- a 'no detection' leads to a failure in case of defect presence and to another inspection in case of defect absence.
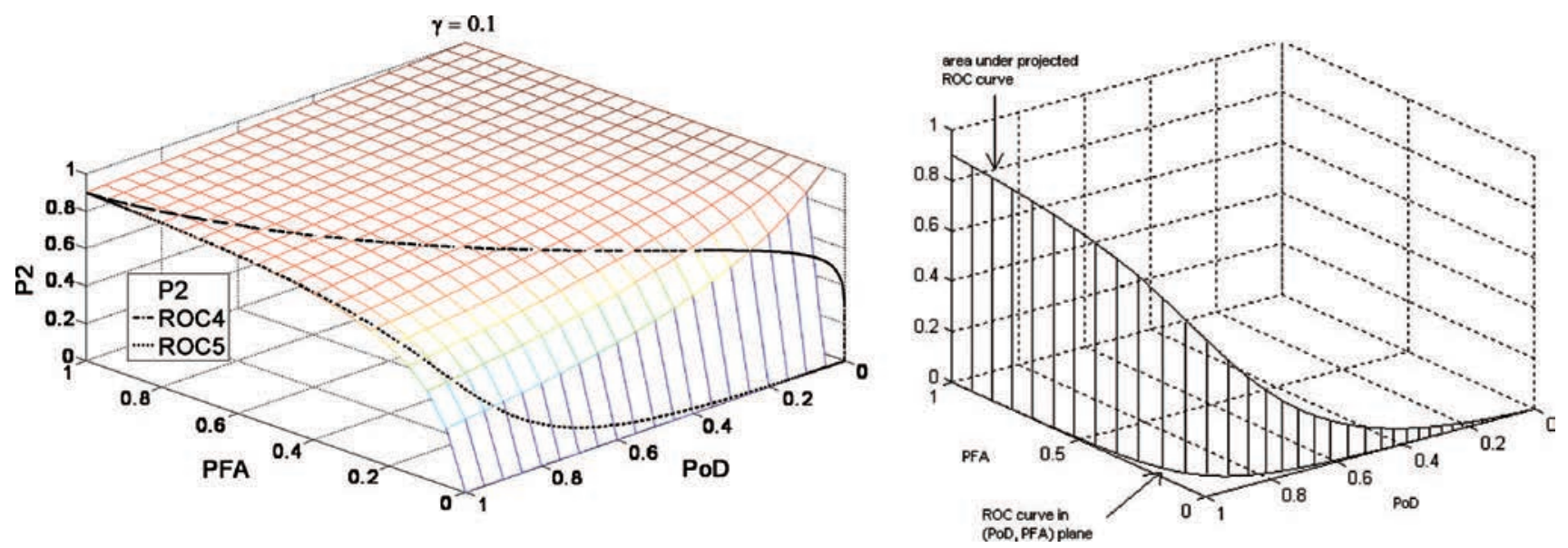

Figure 3. Probability $P_{2}(\gamma=0.1)$ and the area under the projected ROC curve (Schoefs and Clément 2004). 
The subsequent costs are denoted $E(C)_{\mathrm{d}}$ in the case of detection (Equation (13)) and $E(C)_{\text {nd }}$ in the case of no detection (Equation (14)).

$$
\begin{aligned}
& E(C)_{\mathrm{d}}=C_{4}\left(1-P_{2}\right)+C_{2} P_{2} \\
& E(C)_{\mathrm{d}}=C_{4}\left(1-\mu\left(\left(P_{2}\right)_{\mid \mathrm{ROC}}\right)\right)+C_{2} \mu\left(\left(P_{2}\right)_{\mid \mathrm{ROC}}\right) \\
& E(C)_{\mathrm{nd}}=C_{1}\left(1-P_{3}\right)+C_{3} P_{3} \\
& E(C)_{\mathrm{nd}}=C_{1}\left(1-\mu\left(\left(P_{3}\right)_{\mid \mathrm{ROC}}\right)\right)+C_{3} \mu\left(\left(P_{3}\right)_{\mid \mathrm{ROC}}\right)
\end{aligned}
$$

where:

- $C_{1}$ is the cost associated to the event 'no defects knowing that there are no detected defects'. $C_{1}=C_{\text {inspection }}$

- $C_{3}$ is the extra cost associated to the event 'defects knowing that there are no detected defects'. $C_{3}=C_{\text {inspection }}+C_{\text {failure }}$

- $C_{4}$ is the cost associated to the event 'defects knowing that there are detected defects'. $C_{4}=$ $C_{\text {inspection }}+C_{\text {repair }}$

- $C_{2}$ is the extra cost associated to the event 'no defects knowing that there are detected defects'. $C_{2}=C_{\text {inspection }}+C_{\text {repair }}$. Due to the actions defined in the maintenance policy, it is here equal to $C_{4}$.

More complex cost models are described by Breysse et al. (2009) and O'Connor et al. (2008). The probabilities $P_{2}$ and $P_{3}$ are called respectively in the following section CWD (for cost weighting in case of detection) and CWND (for cost weighting in case of no detection) (Schoefs and Clément 2004).

\section{Combination of multiple inspections}

To improve the quality and the reliability of material degradation measurements, the decision-makers can combine and aggregate the results of several techniques of inspection, NDT or destructive testing.

The purpose is to find the combination of inspection techniques which leads to the lowest costs (inspection, repair, failure, etc.). In this article, the ROC curve is supposed to be known for each inspection. For obtaining the resulting ROC curve after multiple inspections, several combination methods can be used. They are listed by Schoefs and Clément (2004). In this study, we use the intersection and union methods given by Yang and Donath (1984). We adopt the condensed expressions presented in Equation (15) for union method and in Equation (16) for intersection method.

$$
\begin{aligned}
& \mathrm{PoD}(1 \cup 2)=\mathrm{PoD}_{1}+\mathrm{PoD}_{2}-\mathrm{PoD}_{1} * \mathrm{PoD}_{2} \\
& \operatorname{PFA}(1 \cup 2)=\mathrm{PFA}_{1}+\mathrm{PFA}_{2}-\mathrm{PFA}_{1} * \mathrm{PFA}_{2}
\end{aligned}
$$

$$
\begin{aligned}
& \operatorname{PoD}(1 \cap 2)=\mathrm{PoD}_{1} * \mathrm{PoD}_{2} \\
& \operatorname{PFA}(1 \cap 2)=\mathrm{PFA}_{1} * \mathrm{PFA}_{2}
\end{aligned}
$$

In practice, the combination of inspection techniques based on the union method allows decreasing the number of missed defects according to the detection threshold. However, the number of false alarms is increasing with this combination technique. On the contrary, the intersection method leads to a higher number of missed defects and a lower number of false alarms, because both of inspection techniques need detecting a defect to take it into account.

In the same way as in part 2.2, a $\operatorname{ROC}(1 \cup 2)$ curve is plotted by connecting points in the plan (PFA $(1 \cup$ $2)$, $\operatorname{PoD}(1 \cup 2))$, and respectively, $\operatorname{ROC}(1 \cap 2)$ curve is plotted by connecting points in the plan (PFA $(1 \cap 2)$, $\operatorname{PoD}(1 \cap 2))$.

\section{Sensibility analysis}

\subsection{Effect of expert's judgement}

Let us now analyse the role of probability of defect presence $\gamma$. It is studied through the sensitivity of $\mu\left(\left(P_{2}\right)_{\mid \text {ROC }}\right)$ to this parameter on Figure 4: low $(\gamma=0.1)$, medium $(\gamma=0.5)$ and high $(\gamma=0.9)$. Several theoretical ROC curves (ROC 3, ROC 4, ROC 6), which are presented in Figure 2, are considered for this study.

Note that $\mu\left(\left(P_{2}\right)_{\mid \mathrm{ROC}}\right)$ of the best NDT tool performance, i.e. ROC 6 curve, is less influenced by the probability of defect presence $\gamma$ than $\mu\left(\left(P_{2}\right)_{\mid \text {ROC }}\right)$ of the worst NDT tool performance, i.e. ROC 4 curve.

When considering $\mu\left(\left(P_{3}\right)_{\mid \mathrm{ROC}}\right)$, the influence of $\gamma$ is very sensitive, but in the opposite sense as for $\mu\left(\left(P_{2}\right)_{\mid \text {ROC }}\right)$ : the geometric mean value of $P_{2}$ is more sensitive for a small probability of defect present $\gamma(0.1-0.5)$ and geometric mean value of $P_{3}$ is more

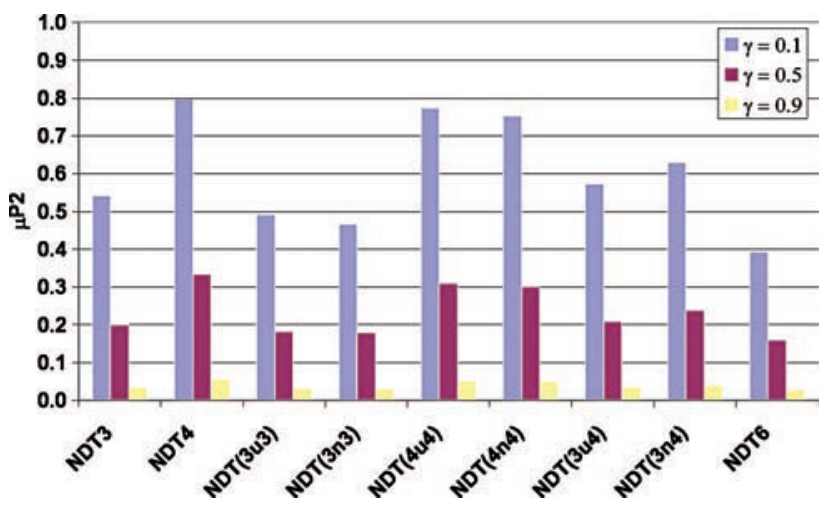

Figure 4. $\mu\left(\left(P_{2}\right)_{\mid \text {ROC }}\right)$ for NDT tools (3) and (4) and resulting from intersection and union methods in the case of a combination of the two NDT tools. 
sensitive for a high probability of defect present $\gamma(0.5-$ $0.9)$.

Consequently, due to the introduction of $\mu\left(\left(P_{2}\right)_{\mid \mathrm{ROC}}\right)$ and $\mu\left(\left(P_{3}\right)_{\mid \mathrm{ROC}}\right)$ in the calculation of cost in case of detection $E(C)_{\mathrm{d}}$ and cost in case of no detection $E(C)_{\text {nd }}$ (Equations (15) and (16)), the probability of defect presence $\gamma$ plays a key role in cost analysis. This aspect will be developed during the following parts of this article.

\subsection{Effect of the combination of multiple inspections}

The objective of this section is to combine several NDT tools by using the intersection and the union methods. Using these two methods allows to analyse the performances after combination and to highlight their interest in terms of cost. The principle of these methods is detailed in Equation (15) for the union method and in Equation (16) for the intersection method.

For illustration, only NDT tools 3 and 4 are used. ROC curves of NDT tools 3 and 4 are presented in Figure 2. Discrepancies between ROC curves can be explained by the technique of measurement and the testing procedure.

All possibilities of combination between NDT tools are used, i.e. either by repeating the same NDT tool or by combining the two different ones. ROC curves resulting from the combination methods are presented in Figure 5. In the figure, the symbols ' $u$ ' and ' $n$ ' indicate an ROC curve obtained by union method or intersection method, respectively.

Results for $\mu\left(\left(P_{2}\right)_{\mid \text {ROC }}\right)$ are presented on Figure 4 for the three levels of $\gamma$ and they confirm the dominant role of the probability of defect presence. Similar results are obtained when considering the $\mu\left(\left(P_{3}\right)_{\mid \text {ROC }}\right)$. It should be reminded that the best performance of NDT tool is the one which minimises $\mu\left(\left(P_{2}\right)_{\mid \text {ROC }}\right)$ and $\mu\left(\left(P_{3}\right)_{\mid \text {ROC }}\right)$, and therefore NDT tool 3 gives better performance than NDT tool 4.

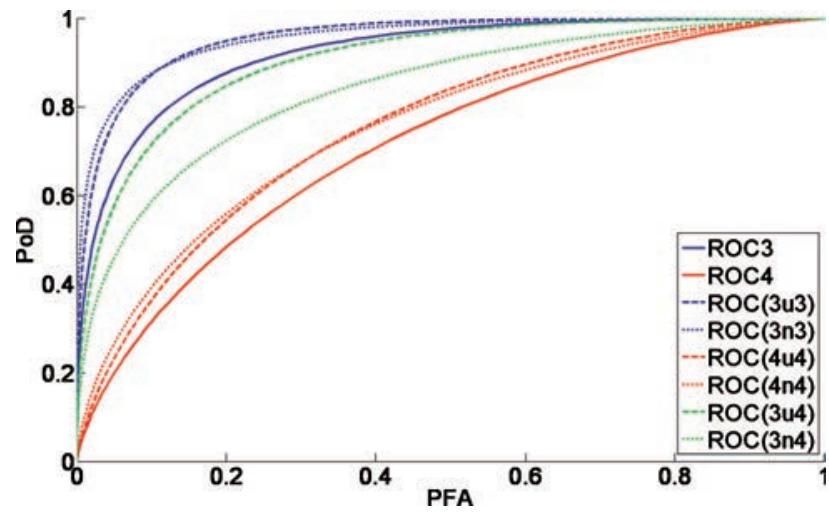

Figure 5. ROC curves resulting from intersection and union methods in the case of a combination of NDT tools (3) and (4).
By combining the same NDT tool, an increase of performance is seen in comparison to the use of the NDT tool only once. This tendency is observed for both NDT tools. Besides, when repeating the same technique, the intersection method is slightly more advantageous for $\mu\left(\left(P_{2}\right)_{\mid \mathrm{ROC}}\right)$ and, on the contrary, the union method gives better results for $\mu\left(\left(P_{3}\right)_{\mid \mathrm{ROC}}\right)$.

The combination of the two different NDT tools gives worse performance than the unique use of the best of both NDT tools. The union method leads to better results for $\mu\left(\left(P_{2}\right)_{\mid \text {ROC }}\right)$ and $\mu\left(\left(P_{3}\right)_{\mid \text {ROC }}\right)$ than intersection method.

Finally, the benefit of performance is more significant where the cases of two different NDT tools are combined.

\subsection{Effect of the cost model}

The objective of this section is to analyse the influence of cost models on the decision process by means of two ratios (Equations (17) and (18)):

$$
\begin{gathered}
C_{a}=\frac{C_{i}}{C_{r}}=\text { 'Cost of inspection Cost of repair' } \\
C_{b}=\frac{C_{r}}{C_{f}}=\text { 'Cost of repair Cost of failure' }
\end{gathered}
$$

Mappings of extra cost expectations in the case of detection $\overline{E(C)_{\mathrm{d}}}$ and in the case of no detection $\overline{E(C)_{\mathrm{nd}}}$ can be plotted for various ranges of the ratio $C_{a}[0.01$; $1.00]$ and $[0.01 ; 0.10]$ for the ratio $C_{b}$. Note that for the study, we fix the value of cost of inspection at 0.01 . These ranges reflect the common values published in the literature that consider the cost of inspection to be generally lower than the cost of repair and that the cost of failure is always higher than the cost of repair. For a value of $C_{b}$ equal to 0.1 , the consequences of failure are weak. In this case, the cost of failure is only equal to the cost of construction or the cost of replacement of a small structure. On the contrary, a value of $C_{b}$ equal to 0.01 corresponds to a structure associated with a high stakes level (security of the persons, availability, etc.).

In the following, we focus on the cost overrun only. From Equations (13) to (14), we consider only the following terms (Equations (19) and (20)):

$$
\begin{aligned}
& \overline{E(C)_{\mathrm{d}}}=C_{2} \mu\left(\left(P_{2}\right)_{\mid \mathrm{ROC}}\right) \\
& \overline{E(C)_{\mathrm{nd}}}=C_{3} \mu\left(\left(P_{3}\right)_{\mid \mathrm{ROC}}\right)
\end{aligned}
$$

Mappings of extra cost expectations in the case of detection $\overline{E(C)_{\text {nd }}}$ are plotted on Figure 6 when $\mu\left(\left(P_{3}\right)_{\mid \text {ROC }}\right)$ equals 0.9 . Extra cost of no detection depends both on ratio $C_{a}$ and ratio $C_{b}$. For higher 
ratios of $C_{a}$ and $C_{b}$, the extra cost of no detection $\overline{E(C)_{\text {nd }}}$ becomes lower. Given that $P_{3}$ is the probability that defects exist knowing that there are no detected defects, a high value of $\mu\left(\left(P_{3}\right)_{\mid \text {ROC }}\right)$ generates an important extra cost of no detection.

Figures of $\overline{E(C)_{\mathrm{d}}}$ are not plotted. It is shown that this extra cost of detection depends only on ratio $C_{a}$. When the ratio 'Cost of inspection/Cost of repair' increases, the cost of detection $\overline{E(C)_{\mathrm{d}}}$ decreases. Given that $P_{2}$ is the probability that there are no defects knowing that there are detected defects, a high value of $\mu\left(\left(P_{2}\right)_{\mid \text {ROC }}\right)$ generates a significant extra cost in case of detection.

Finally, for most cases in the civil engineering field, the extra cost of no detection is more severe than the extra cost of detection. This is because stakes associated with infrastructure are very important by comparison with maintenance costs (inspection and repair).

\section{Parametric modelling: effect of the shape of ROC curves on the decision process: the $\alpha \delta$ method}

\subsection{Aim and principle}

A simple geometric characterisation of ROC curves is the distance between the curve and the BPP of coordinates $(\mathrm{PFA}=0, \mathrm{PoD}=1)$ (Schoefs and Clément 2004): by definition, the greater the distance, the worst the performance. The corresponding point on the ROC curve is called the performance point of the NDT tool (NDT-BPP). However, as the configurations of ROC curves for the same distance are varied, we in this article explain this measure of performance by using the polar coordinates of the NDT-BPP. The $\alpha \delta$ method lies on this characterisation. The NDT-BPP polar coordinates are then defined by:

- the radius $\delta_{\mathrm{NDT}}$ is the performance index (NDTPI) (distance between the BPP and the ROC

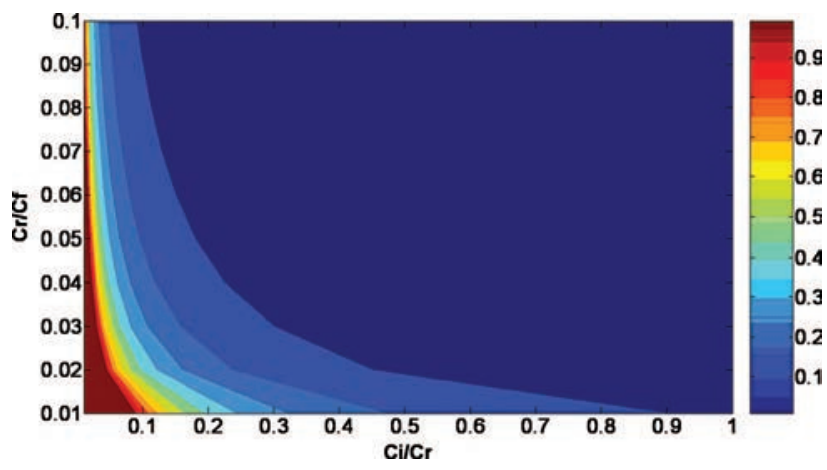

Figure 6. Mapping of extra cost expectation in case of no detection $\overline{E(C)_{\text {nd }}}$ when $\mu\left(\left(P_{3}\right)_{\mid \text {ROC }}\right)$ equals 0.9 . curve) (Schoefs and Clément 2004, Schoefs et al. 2007);

- the angle $\alpha_{\mathrm{NDT}}$ between axis $(\mathrm{PFA}=0)$ and the line (BPP, NDT-BPP).

The objective of the $\alpha \delta$ method is to perform parametric studies lying on two parameters to analyse the effect of the shape of ROC curves on the decision process relating to IMR. To achieve this goal, the influence of the performance of ROC curves, represented by $\delta_{\mathrm{NDT}}$ and $\alpha_{\mathrm{NDT}}$, is appreciated through the costs $\overline{E(C)_{\mathrm{d}}}$ and $\overline{E(C)_{\mathrm{nd}}}$.

In order to perform this parametric study, we assume that 'signal + noise' and 'noise' amplitude are normally distributed: $N\left(\mu_{\mathrm{s}+\mathrm{n}}, \sigma_{\mathrm{s}+\mathrm{n}}\right)$ and $N\left(0, \sigma_{\mathrm{n}}\right)$. To simulate a set of couples ( $\left.\delta_{\mathrm{NDT}}, \alpha_{\mathrm{NDT}}\right)$ we modify the values of $\mu_{\mathrm{s}+\mathrm{n}}, \sigma_{\mathrm{s}+\mathrm{n}}$ and $\sigma_{\mathrm{n}}$. Values of the mean $\mu_{\mathrm{s}+\mathrm{n}}$ stay in the range $[0 ; 10]$, when standard deviations $\sigma_{\mathrm{s}+\mathrm{n}}$ and $\sigma_{\mathrm{n}}$ both belong to $[0.5 ; 5]$. We modify these values with a step of 0.5 .

Polar coordinates of the BPP of the NDT tool (NDT-BPP) are computed for each set of pdf parameters.

\subsection{Analysis of the NDT-BPP data base}

Figure 7 presents the mapping of NDT-BPP in the plan (PFA, PoD). We notice that all of the NDT-BPP have coordinates between 0.4 and 1.0 for $\mathrm{PoD}$ and between 0.0 and 0.6 for PFA: that represents an interesting area regarding the analysis of the effect that the shape of ROC curves has on the decision process.

Four examples of NDT tools performances represented by ROC curves are plotted on Figure 7. For each one, Cartesian and polar coordinates of NDTBPP are given in Table 1. NDT-BPP1 corresponds to the worst NDT tool performance, meaning that some noise can be easily detected, even if nothing is to be

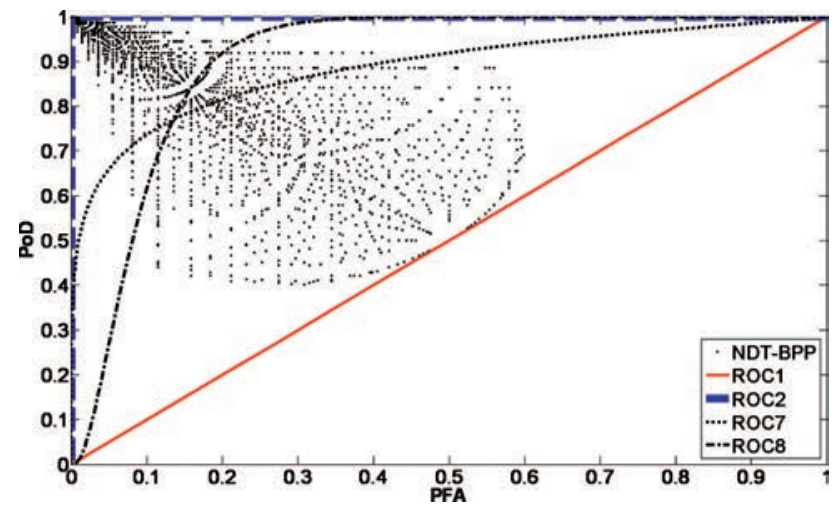

Figure 7. Data base of NDT-BPP plotted in Cartesian plane (PFA, PoD). 
detected (no defect presence), which finally leads to a high number of false alarms. On the other hand, NDTBPP2 represents the best NDT tool performance in our numerical data-base. In this case, the PoD reaches very quickly to values near 1 , with small probabilities of false alarms for high values of probabilities of detection.

The performances of NDT tools 1 and 2 can be easily compared with the radius $\delta_{\mathrm{NDT}}$, i.e. 0.71 for NDT-BPP1 versus 0.00 for NDT-BPP2. But between these two extreme levels of NDT tools performances, there is a multitude of cases where this single parameter does not allow for differentiating ROC curves. To illustrate the role of $\alpha_{\mathrm{NDT}}$, the maintenance policy and the extra cost functions are the same as those described in the previous section. The cost model selected for the application is given on Table 2.

The best of the four NDT tools is the one which minimises the two extra cost functions or a combination of them (sum, etc.), related to the cases of detection or no detection. To complete the parametric study, we also consider three values for the probability of defect presence $\gamma$, i.e. $0.1,0.5$ and 0.9 , that is needed when computing probabilities that govern the extra cost expectation.

The results of cost analysis are presented in Table 3. Let us focus on the comparison between NDT tools 7 and 8 . It is interesting to notice that for the three values of $\gamma$, the extra cost in case of detection $\overline{E(C)_{\mathrm{d}}}$ is always minimum for NDT tool 7 and the extra cost of no detection $\overline{E(C)_{\text {nd }}}$ is always minimum for NDT tool 8. Here, the extra cost in case of no detection is much larger than the extra cost in case of detection. It comes clearly from the selected maintenance policy and cost

Table 1. Cartesian and polar coordinates of NDT-BPP.

\begin{tabular}{lccccr}
\hline & \multicolumn{2}{c}{$\begin{array}{c}\text { Cartesian } \\
\text { coordinate }\end{array}$} & & \multicolumn{2}{c}{$\begin{array}{c}\text { Polar } \\
\text { coordinate }\end{array}$} \\
\cline { 2 - 3 } \cline { 5 - 6 } & PFA & PoD & & $\delta$ & $\alpha\left(^{\circ}\right)$ \\
\hline NDT-BPP1 & 0.50 & 0.50 & & 0.71 & 45.00 \\
NDT-BPP2 & 0.00 & 1.00 & & 0.00 & 00.00 \\
NDT-BPP7 & 0.16 & 0.79 & & 0.26 & 36.83 \\
NDT-BPP8 & 0.18 & 0.88 & & 0.21 & 57.23 \\
\hline
\end{tabular}

Table 2. Cost model selected for failure, repair, inspection and inspection combination.

\begin{tabular}{lc}
\hline & Cost \\
\hline Failure & 1.000 \\
Repair & 0.010 \\
Inspection & 0.001 \\
Inspection combination & 0.002 \\
\hline
\end{tabular}

models. With another cost model, this tendency can be reversed (O'Connor et al. 2008, Breysse et al. 2009). In this case, the radius $\delta_{\mathrm{NDT}}$ is no longer sufficient to characterise the performance of NDT tools and it is necessary to use the two polar coordinates: the radius $\delta_{\mathrm{NDT}}$ and the angle $\alpha_{\mathrm{NDT}}$.

This last point is confirmed by the fact that simulated values of extra costs of detection and extra costs of no detection according to $\delta_{\mathrm{NDT}}$ are significant. Results for $\overline{E(C)_{\mathrm{d}}}$ are presented in Figure 8 for three probabilities of defect presence $\gamma$. This sensitivity is observed for $\overline{E(C)_{\text {nd }}}$ too.

This figure shows that for a given $\delta_{\mathrm{NDT}}$, the scatter in extra cost expectation is very sensitive to $\gamma$. When considering $\alpha_{\mathrm{NDT}}$, similar results are obtained but the sensitivity is not the same. As for the radius $\delta_{\mathrm{NDT}}$, the angle $\alpha_{\text {NDT }}$ cannot be used alone to characterise the performance of an NDT tool. Consequently, the following study is dedicated to analyse extra costs of detection and extra costs of no detection according to the whole numerical data-base of NDT-BPP.

\subsection{Influence of NDT-BPP polar coordinates on extra cost of detection and extra cost of no detection}

For illustration, Figure 9 presents mappings of extra cost of no detection according to the data-base of

Table 3. Performances of the four NDT tools based on cost analysis.

\begin{tabular}{lcccccccc}
\hline & \multicolumn{2}{c}{$\gamma=0.1$} & & \multicolumn{2}{c}{$\gamma=0.5$} & & \multicolumn{2}{c}{$\gamma=0.9$} \\
\cline { 2 - 3 } & $\overline{E(C)_{\mathrm{d}}}$ & $\overline{E(C)_{\mathrm{nd}}}$ & $\overline{E(C)_{E}}$ & $\overline{E(C)_{\mathrm{nd}}}$ & & $\overline{E(C)_{\mathrm{d}}}$ & $\overline{E(C)_{\mathrm{nd}}}$ \\
\hline NDT1 & 0.010 & 0.100 & & 0.006 & 0.501 & & 0.001 & 0.901 \\
NDT2 & 0.004 & 0.026 & & 0.002 & 0.154 & & $3.10^{-4}$ & 0.372 \\
NDT7 & 0.006 & 0.040 & & 0.002 & 0.252 & & $4.10^{-4}$ & 0.713 \\
NDT8 & 0.008 & 0.032 & & 0.003 & 0.189 & & $5.10^{-4}$ & 0.457 \\
\hline
\end{tabular}

Note: Italicised values are the minimum value for each value of $\gamma$ and each NDT.

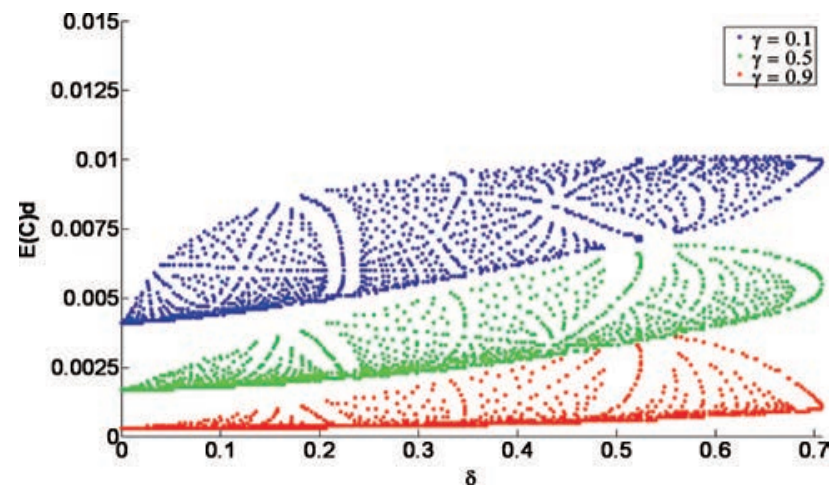

Figure 8. Simulated values of extra costs of detection $\overline{E(C)_{\mathrm{d}}}$ according to the radius $\delta_{\mathrm{NDT}}$. 
NDT-BPP in polar $\left(\delta_{\mathrm{NDT}}, \alpha_{\mathrm{NDT}}\right)$ coordinates, for $\gamma=0.9$.

For this large probability of defect presence $(\gamma=0.9)$, extra costs of no detection for $\gamma=0.9$ increase when $\alpha_{\mathrm{NDT}}$ decreases and $\delta_{\mathrm{NDT}}$ increases. Note that when considering extra costs of detection, they are maximum in the zone defined by values of $\delta_{\text {NDT }}$ higher than 0.3 and $\alpha_{\text {NDT }}$ higher than $50^{\circ}$. Outside this area, extra cost of detection increases mainly with $\delta_{\text {NDT }}$.

For a low probability of defect presence $(\gamma=0.1)$, other results have been obtained: extra costs of detection are minimum when associated mainly to values of radius $\delta_{\mathrm{NDT}}$ lower than 0.4 and values of angle $\alpha_{\mathrm{NDT}}$ lower than $40^{\circ}$. Maximum extra costs of detection are located in zone with high values of $\delta_{\mathrm{NDT}}$ and $\alpha_{\mathrm{NDT}}$. It is also interesting to note that for values of $\delta_{\mathrm{NDT}}$ lower than 0.1 , the influence of $\alpha_{\mathrm{NDT}}$ is negligible. In addition, extra costs of no detection for $\gamma=0.1$ are maximum for values of $\delta_{\mathrm{NDT}}$ superior than 0.1 and for values of $\alpha_{\mathrm{NDT}}$ minor than $30^{\circ}$. When the values of $\alpha_{\mathrm{NDT}}$ are higher than $30^{\circ}$, the extra costs of no detection increase mainly as a function of $\delta_{\mathrm{NDT}}$.

Now, three stake levels are considered: high, medium and low. For each one, a cost model is defined (see Table 4). Previous correspond to a cost model associated with a high stakes level. For the three examples of cost models, mappings of extra cost of detection are the same because the cost of inspection

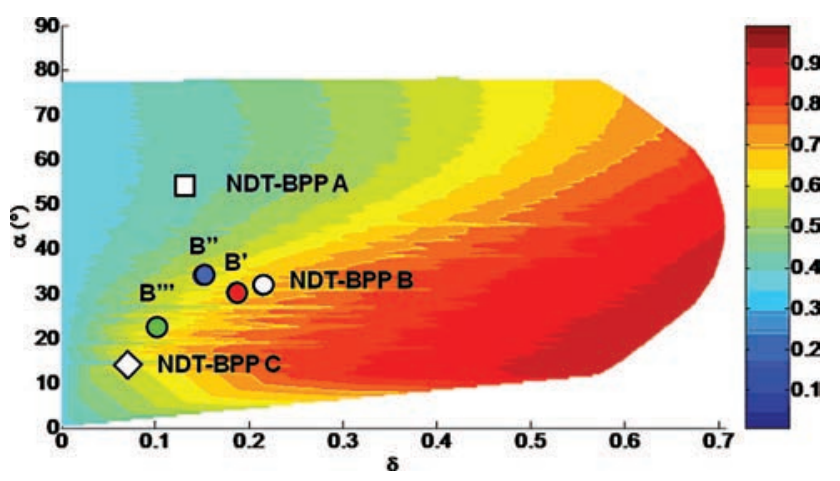

Figure 9. Mapping of extra cost of no detection $\overline{E(C)_{\mathrm{nd}}}$ in polar plane for $\gamma=0.9$.

Table 4. Cost models associated with several stake levels.

\begin{tabular}{llll}
\hline Stakes & High & Medium & Low \\
\hline Cost of failure, $C_{\mathrm{f}}$ & 1.0 & 0.5 & 0.1 \\
Cost of repair, $C_{\mathrm{r}}$ & 0.01 & 0.01 & 0.01 \\
Cost of inspection, $C_{\mathrm{i}}$ & 0.001 & 0.001 & 0.001 \\
$C_{\mathrm{i}} / C_{\mathrm{r}}$ & 0.1 & 0.1 & 0.1 \\
$C_{\mathrm{r}} / C_{\mathrm{f}}$ & 0.01 & 0.02 & 0.1 \\
\hline
\end{tabular}

and the cost of repair are similar in each case. Therefore, only mappings of extra cost of no detection according to Cartesian and polar coordinates of NDTBPP are presented for $\gamma$ equal to 0.9 (see Figure 10).

The results from these figures show that the extra cost of no detection decreases when the stakes level decreases.

Consequently, for structures associated with a low stakes level and for low probability of defect presence $(\gamma=0.1)$, extra cost of detection and extra cost of no detection are similar. In this case, the two polar coordinates, radius $\delta_{\mathrm{NDT}}$ and angle $\alpha_{\mathrm{NDT}}$, are essential to characterise the performance of NDT tools.

\subsection{Use of NDT-BPP polar coordinates for NDT-tool improvement in RBI context}

Let us now consider the risk-based improvement of a NDT tool. We assume that only the noise can be modified. The question is: 'How much should I reduce the noise (i.e. the standard deviation of its distribution) to reach a given level of risk?' It is equivalent to search the reduction factor $\rho(0<\rho<1)$ applied on the standard deviation that allows to reduce the cost expectation.

Let us start from the situation of NDT-BPP B. By using reduction factors $0.8,0.6$ and 0.4 , the displacement of this performance point is illustrated on Figure 9 by the position of the points NDT-BPP $\mathrm{B}^{\prime}, \mathrm{B}^{\prime \prime}$ and $\mathrm{B}^{\prime \prime \prime}$, respectively. This figure illustrates the interest of the $\alpha \delta$ method and the associated graphical representation: the effect of the reduction factor is not linear and moreover the trajectory of the NDT-BPP is not linear. As a perspective, the cost of inspection improvement can be now easily introduced.

\section{Combination inspections: example of application for the RBI of steel harbour structures}

\subsection{Context}

The objective of this section is to analyse the combination effect when repeating the same or different NDT tools (i.e. same ROC) twice. The article focuses on the combination of two inspections in view of simplifying the analysis of the results. However, the method can be extended to multiple inspections without any difficulty. The role of expert judgement on the minimisation of the extra cost expectation and on the ranking decisions is also analysed. For the combination, we consider a memory-less inspection: the inspection is carried out by two inspectors who do not remember the previous result at the same location. Moreover, we mention that inspections are performed in harsh conditions. The result depends mainly on a set of human factors such as the experience and the 
tiredness of the inspector. Then, the results of inspections from the two inspectors are considered as independent events. Equations (15) and (16) can then be used.

The methodology is illustrated for the RBI of steel harbour structures submitted to uniform corrosion (steel sheet pile seawalls). In this example, we consider only the results provided by ultrasonic residual thickness measurements, which is a common technique for identifying the zones on steel marine structures that are the most affected by corrosion.

ROC curves obtained for two wharves by two couples (diver-inspection society) for the same level of corrosion are presented in Figure 11 (ROC B and C). ROC curves are plotted by discrete distributions of 'signal + noise' and 'noise'. For simplicity, each couple is named NDT tool in the following sections.

The parameters of the $\alpha \delta$ method (polar coordinates of NDT-BPP) for the three NDT tools are indicated on mappings of the extra cost of detection and extra cost of no detection which correspond to the same cost model as that used in this application. A comparison between results given by mappings and cost analysis carried out during this application is also suggested in the following section.

On the basis of analysis of $\delta_{\mathrm{NDT}}$ only, ROC C curve represents the best performance of the three different NDT tool results and the ROC B curve is the worst. Measurements are performed in the immersion area for which the inspection conditions are harsh (Schoefs et al. 2008b).

\subsection{Combination of inspections}

The objective of this section is to combine characteristics of ROC obtained from different couples (diverinspection society).

ROC curves obtained by intersection and union methods of NDT tool results B and NDT tool results $\mathrm{C}$ when repeating the same NDT tool are represented in Figure 11a, and on Figure 11b when repeating several NDT tools.
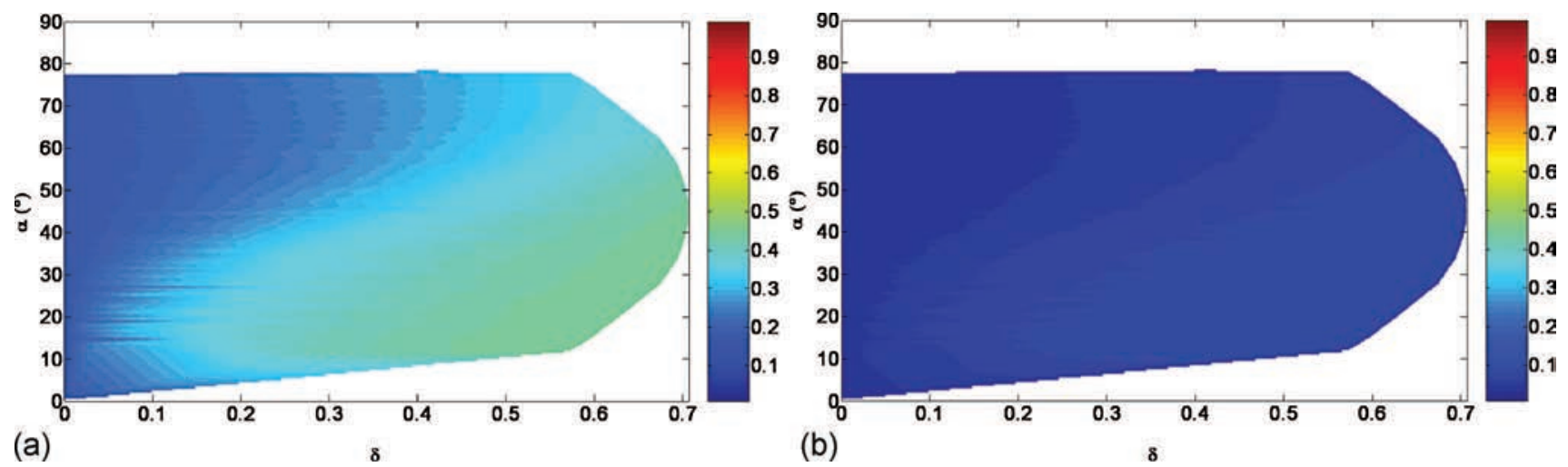

Figure 10. Mapping of extra cost of no detection $\overline{E(C)_{\text {nd }}}$ in polar plane for $\gamma=0.9$ and cost model associated with medium (a) and low (b) stakes levels.
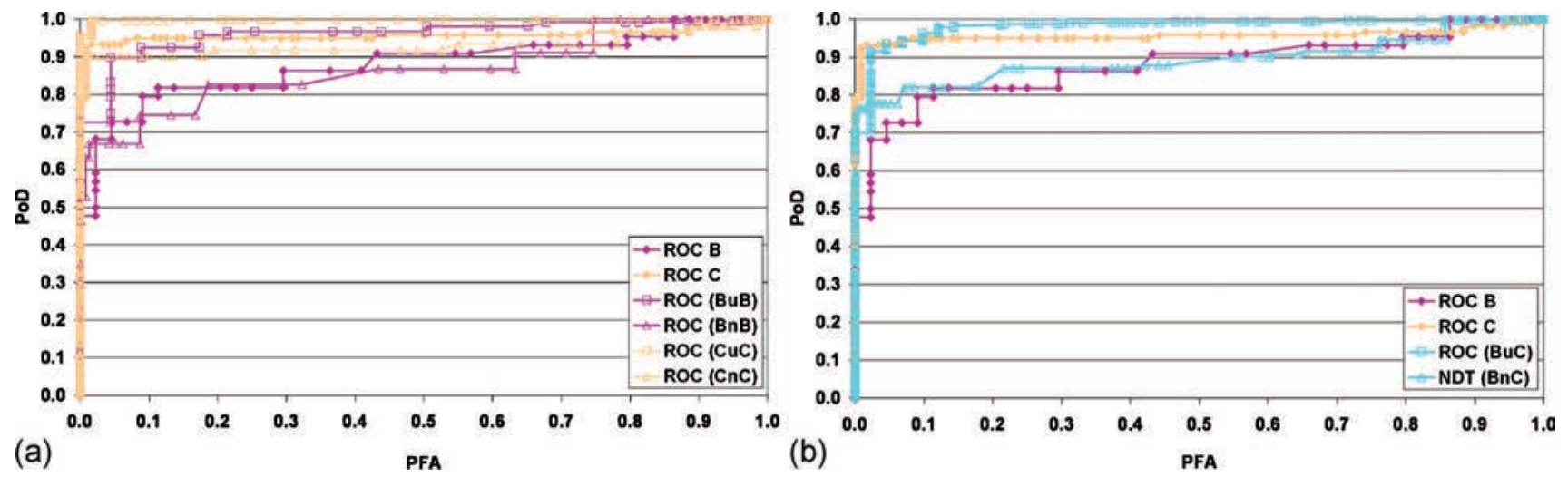

Figure 11. (a) ROC curves resulting from intersection and union methods for NDT tools B and C when repeating the same NDT tools and (b) when using the two inspection tools. 

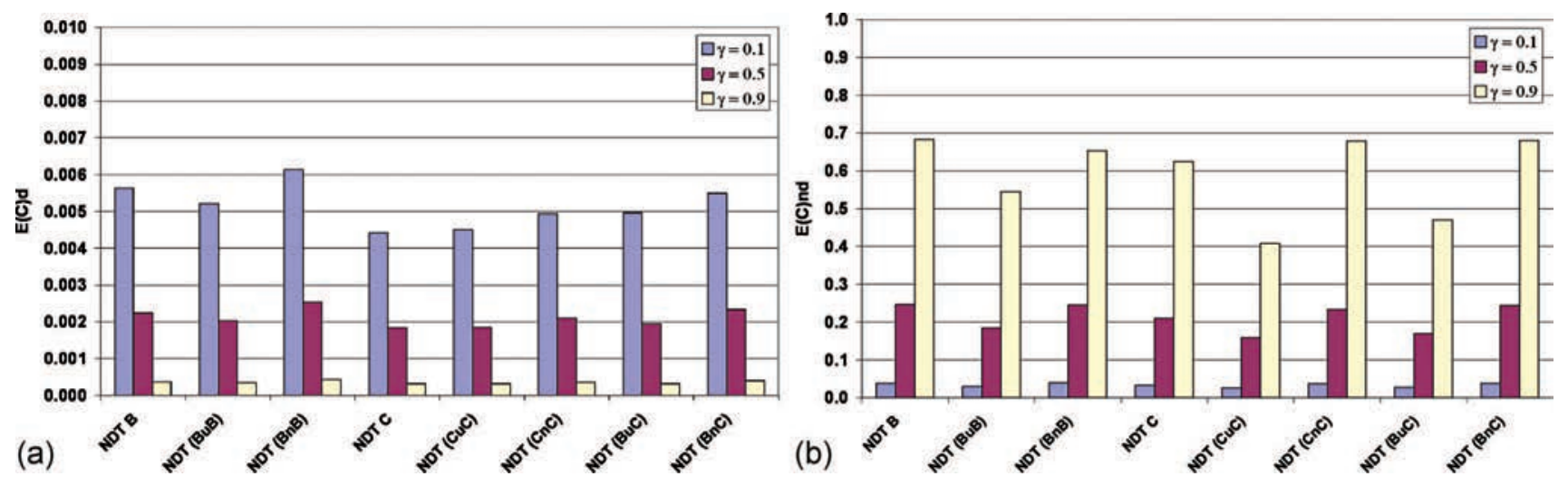

Figure 12. Influence of $\gamma$ on the extra cost in the case of detection $\overline{E(C)_{\mathrm{d}}}$ (a) and no detection $\overline{E(C)_{\mathrm{nd}}}(\mathrm{b})$.

\subsection{Sensitivity of cost analysis to expert judgement}

In the following, we focus on the two choices that affect the extra costs in our methodology both in the case of detection $\overline{E(C)_{\mathrm{d}}}$ and in the case of no detection $\overline{E(C)_{\mathrm{nd}}}$ :

- the probability of defect presence $\gamma$

- the decision after two inspections (union or intersection methods).

The results are presented in Figure 12. The extra cost in case of no detection is much larger than extra cost in case of detection. This can be clearly seen from the selected cost model (huge discrepancy between $C_{\text {repair }} / C_{\text {failure }}$ ratio and $C_{\text {inspection }} / C_{\text {repair }}$ ratio).

When analysing $\mu\left(\left(P_{2}\right)_{\mid \text {ROC }}\right)$ and $\mu\left(\left(P_{3}\right)_{\mid \text {ROC }}\right)$, it has been noticed that union method leads to better performances than intersection method in all cases of combination. In contrary to the theoretical study (section 5.2.), performances obtained after combining the same NDT tool by intersection method are always worse than when the NDT tool is used once. Furthermore, when combining different techniques, union method gives better performance than the use of only the best of both NDT tools. This analysis shows the interest of repeating the same or a different technique (Schoefs and Clément 2004) but also enables to the influence of expert's judgement on the choice of the combination method to be seen. The expert faces two decisions: there is a defect if both NDT tools give a defect or if one of them gives this result.

Let us now analyse the role of the probability of defect presence $\gamma$ in three cases: low $(\gamma=0.1)$, medium $(\gamma=0.5)$ and high $(\gamma=0.9)$ values. Influence of $\gamma$ is in an opposite way for $\overline{E(C)_{\mathrm{d}}}$ and $\overline{E(C)_{\mathrm{nd}}}$ : the extra cost of detection is more important for small probabilities of defect presence $(0.1-0.5)$ and the extra cost of no detection is more important for high probabilities of defect presence $(0.5-0.9)$. It comes from the fact that
Table 5. Performances of the three NDT tools (A, B \& C) based on cost analysis.

\begin{tabular}{lccccc}
\hline & \multicolumn{2}{c}{$\gamma=0.1$} & & \multicolumn{2}{c}{$\gamma=0.9$} \\
\cline { 2 - 3 } \cline { 5 - 6 } & $\overline{E(C)_{\mathrm{d}}}$ & $\overline{E(C)_{\mathrm{nd}}}$ & & $\overline{E(C)_{\mathrm{d}}}$ & $\overline{E(C)_{\mathrm{nd}}}$ \\
\hline NDT B & 0.006 & 0.039 & & $4.10^{-4}$ & 0.682 \\
NDT C & 0.004 & 0.033 & & $3.10^{-4}$ & 0.624 \\
\hline
\end{tabular}

Table 6. Cartesian and polar coordinates of the three NDT$\mathrm{BPP}(\mathrm{A}, \mathrm{B} \& \mathrm{C})$.

\begin{tabular}{lllllr}
\hline & \multicolumn{2}{c}{$\begin{array}{c}\text { Cartesian } \\
\text { coordinate }\end{array}$} & & \multicolumn{2}{c}{$\begin{array}{c}\text { Polar } \\
\text { coordinate }\end{array}$} \\
\cline { 2 - 3 } \cline { 5 - 6 } & PFA & PoD & & $\delta$ & $\alpha\left(^{\circ}\right)$ \\
\hline NDT-BPPB & 0.11 & 0.82 & & 0.21 & 32.00 \\
NDT-BPPC & 0.02 & 0.93 & & 0.07 & 14.06 \\
\hline
\end{tabular}

$\overline{E(C)_{\mathrm{d}}}$ and $\overline{E(C)_{\text {nd }}}$ depend respectively on $\mu\left(\left(P_{2}\right)_{\mid \text {ROC }}\right)$ and $\mu\left(\left(P_{3}\right)_{\mid \mathrm{ROC}}\right)$. We remind that $P_{2}$ is the probability of no defect presence, conditional to defect detection and $P_{3}$ is the probability of defect presence, conditional to no defect detection. So, $P_{2}$ is directly associated with false alarms, and $P_{3}$ with no detections. False alarms are more sensitive when probabilities of defect presence are small. On the other hand, no detections are more sensitive when probabilities of defect presence are high.

Furthermore, extra costs due to detection and no detection increase when performances of ROC curves decrease. To illustrate this comment, we compare extra costs obtained in this study for the NDT tools B and C (see Table 5) and the mappings where parameters of the corresponding $\alpha \delta$ method (polar coordinates of NDT-BPP) were indicated (see Figure 9). Besides, polar and Cartesian coordinates of the two NDT-BPP (B \& C) are detailed in the Table 6. NDT-A is not considered in this article. 
We highlight the fact that extra costs of detection and no detection obtained during this illustration are coherent with mappings for NDT-B and NDT-C. This is because of the sensibility of extra costs to the polar coordinates of NDT-BPP. Indeed, the lack of precision on the value of $\alpha_{\mathrm{NDT}}$ when discrete distributions are used, can lead to a bad exploitation of the previous mappings. To increase the reliability to characterise the 'noise' and 'signal + noise', we suggest to use the projection on polynomial chaos (Schoefs 2009, Schoefs et al. 2008b, 2009).

\section{Conclusion}

Concepts of ROC curves coming from detection theory are very useful to quantify the performance of nondestructive techniques. This article reviews the theoretical aspects coming from detection theory and probabilistic modelling of inspections results with the view to provide inputs in the computation of mathematical expectation of RBI cost models. Methods of combination of multiple inspections are also presented.

Sensibility analysis shows the influence of inputs on RBI concepts. Effects of expert judgement and cost model are highlighted. The effect of the shape of ROC curves on the extra costs is analysed through the $\alpha \delta$ method. The objective of the parametric study is to assess the influence of the performance of NDT tools, characterised by polar coordinates $\delta_{\mathrm{NDT}}$ and $\alpha_{\mathrm{NDT}}$ in the plane (PFA, PoD), through the expectation of the extra costs in the case of detection $\overline{E(C)_{\mathrm{d}}}$ and in the case of non-detection $\overline{E(C)_{\mathrm{nd}}}$.

Finally, this article shows the interest of combination methods, i.e. intersection or union, for highlighting the impact of the expert judgement in the decision process at two levels:

- the way to address the decision after obtaining results from the two inspections (choice of combination methods);

- the estimation of the probability of defect existence that is needed when computing the probability of events that govern the extra cost expectation.

These aspects are treated by an illustration of the process for the RBI of steel harbour structures submitted to uniform corrosion.

\section{References}

Barnouin, B., et al., 1993. Underwater inspection reliability trials for offshore structures. Proceedings of the 12th international conference on offshore mechanics and arctic engineering, Vol. 2. NY: ASME, 883-890.
Breysse, D., et al., 2009. Life cycle cost analysis of ageing structural components based on non destructive condition assessment. Australian Journal of Structural Engineering, 9 (1), 55-66. ISSN: 1328-7982.

O'Connor, A., et al., 2008. Disaster and hazard mitigation for infrastructural elements/networks through provision of a two stage inspection process. In: Proceedings of the third international forum on engineering decision making, 'Optimal strategies for disaster and hazard mitigation', IFED, 12-15 December 2007, Port Stephens, New South Wales, Australia.

Pakrashi, V., et al., 2010. ROC dependent event isolation method for image processing based assessment of corroded harbour structures. Structure and Infrastructure Engineering: Maintenance, Management, Life-Cycle Design and Performance, 6 (3), 365-378.

Rouhan, A., 2001. Structural integrity evaluation of existing offshore platforms based on inspection data. Thesis (PhD). University of Nantes, France.

Rouhan, A. and Schoefs, F., 2003. Probabilistic modelling of inspections results for offshore structures. Structural Safety, 25, 379-399.

Rudlin, J.R., 1996. Reliability of inspection for fatigue cracks in offshore structures. Savoy Place, London, UK: Institution of Electrical Engineers, 6/1-6/3.

Rudlin, J.R. and Dover, W.D., 1996. The ICON Projectdata for Underwater Inspection. Insight, 38 (6), 412-414.

Schoefs, F., 2009. Risk analysis of structures in presence of stochastic fields of deterioration: coupling of inspection and structural reliability. Australian Journal of Structural Engineering, Special Issue "Disaster \& Hazard Mitigation", 9 (1), 67-78.

Schoefs, F. and Clément, A., 2004. Multiple inspection modeling for decision making and management of jacket off-shore platforms: effect of false alarms. In: Proceedings of the 1st international forum on engineering decision making (IFED'04), 5-9 December 2004, Stoos, Switzerland.

Schoefs, F., et al., 2007. Spatial dependence of receiver operating characteristic curves for risk based inspection of corroded structures: application to on-pile wharf. In: J. Kanada, T. Takada, and H. Furuta, eds. Proceedings of 10th international conference on applications of statistics and probability in civil engineering (I.C.A.S.P'07). July 31-August 3 2007, Tokyo, Japan. ISBN 978-0-41545134-5.

Schoefs, F., et al., 2008a. Expert judgement for combining NDT tools in RBI context: application to marine structures. In: 4th ASRANet Colloquium, 25-27 June 2008, Athens, Greece, ASRANet Ltd.

Schoefs, F., et al., 2008b. Inspection of steel structures in coastal area with NDT tools: use of polynomial chaos decomposition for building receiver operating curves. In: Proceeding of 1st international conference on applications heritage and constructions in coastal and marine environment, (MEDACHS'08), 28-30 January 2008, Lisbon, Portugal: LNEC.

Schoefs, F., Clément, A., and Nouy, A., 2009a. Assessment of spatially dependent ROC curves for inspection of random fields of defects. Structural Safety, 31 (5), 409419.

Schoefs, F., et al., 2009b. Optimal maintenance planning of randomly ageing serial networks based on the assessment of the number of failed components. Computer Aided Civil and Infrastructure Engineering, 24 (7), 492-508. 
Sheils, E., et al., 2008. Development of a two stage inspection process for the assessment of deteriorating bridge structures. Reliability Engineering and System Safety, 95, 182-194.

Straub, D. and Faber, M.H., 2003. Modelling dependency in inspection performance. In: Madanat and Pestana, eds. Proceedings of application of statistics and probability in civil engineering, ICASP 2003, San Francisco, Der Kiureghian. Rotterdam: Millpress, 1123-1130. ISBN 90 59660048
Yang, J.R. and Donath, R.C., 1984. Improving NDE capability through multiple inspection with application to gas turbine engine disks. Report Number: AFWAL TR $82-4111,85 \mathrm{p}$. 